\title{
Signo de la copa de vino: A propósito de un caso con esclerosis lateral primaria y demencia frontotemporal.
}

\author{
Wine glass sign in a patient with primary lateral sclerosis and frontotemporal dementia: a case report. \\ Darwin Segura-Chávez ${ }^{1, a}$, Luis Torres-Ramírez 1a,b, Miriam Vélez-Rojas ${ }^{1 a, b}$, Martha Flores-Mendoza ${ }^{1, a, b}$. \\ RESUMEN
}

La esclerosis lateral primaria es muy poco frecuente, representa 2-4 \% del grupo de enfermedades de motoneurona. Se caracteriza por espasticidad corticoespinal y síndrome pseudobulbar. La resonancia magnética nuclear muestra lesiones hiperintensas de secuencias potenciadas en T2; cuando el compromiso es bilateral, la imagen da la apariencia de "copa de vino". Caso clínico: Paciente varón de 56 años de edad, con un cuadro clínico de dos años de evolución, caracterizado por desinhibición, apatía, y conducta social inapropiada. La evolución clínica mostró criterios diagnósticos de esclerosis lateral primaria y de demencia frontotemporal. La resonancia magnética de encéfalo, protocolo T2, corte coronal reveló el compromiso bilateral de los haces cortico-espinales desde el centro semioval hasta las pirámides bulbares semejando la imagen en "copa de vino". Aunque infrecuente, el caso muestra la posible asociación de Esclerosis Lateral Primaria (ELP) con demencia frontotemporal; la imagen en "copa de vino" puede estar presente en enfermedad de motoneurona y, cuando particularmente asociada a ELP, puede ser de gran ayuda en el diagnostico diferencial con otras entidades crónicas de curso clínico similar.

PALABRAS CLAVE: Demencia frontotemporal, enfermedad de neurona motora, esclerosis lateral primaria, Signo de la "copa de vino"

\section{SUMMARY}

Primary lateral sclerosis is a very uncommon progressive disease and represents $2-4 \%$ of motor neuron diseases group. It is characterized by cortico-spinal spasticity and pseudo bulbar syndrome. Magnetic resonance shows white matter hyperintensities in $\mathrm{T} 2$ weight sequence. When the impairment is symmetrical, the image takes the appearance of a "wine glass". Clinical case: A 56-year-old male patient with a clinical picture of 2-years' duration characterized by disinhibition, apathy, and inappropriate social behavior. The clinical evolution met the diagnostic criteria of primary lateral sclerosis and frontotemporal dementia. Magnetic resonance imaging in coronal T2 weighted sequences, showed symmetrical impairment of corticospinal pathway from the semiovale centrum to the medullary pyramids showing the "wine glass" appearance. Although infrequent, the case shows the possible association of Primary Lateral Sclerosis (PLS) with frontotemporal dementia; the "wine glass" image in magnetic resonance may be present in motor neuron disease, when particularly associated with PLS may be of great help in the differential diagnosis with other chronic entities of similar clinical course.

KEY WORDS: Frontotemporal dementia, motor neuron disease, primary lateral sclerosis, "Wine glass" appearance

1 Departamento de Enfermedades Neurodegenerativas, Instituto Nacional de Ciencias Neurológicas. Lima, Perú.

a Médico Neurólogo; b Departamento de Enfermedades Neurodegenerativas. 
Signo de la copa de vino: A propósito de un caso con esclerosis lateral primaria y demencia frontotemporal.

\section{INTRODUCCIÓN}

La esclerosis lateral primaria (ELP) está considerada dentro del grupo de enfermedades de motoneurona, junto con la atrofia muscular espinal y la esclerosis lateral amiotrófica (ELA), es la menos común de las enfermedades de motoneurona (EMN) representa $2-4 \%$ de estas (1).

Se considera una enfermedad progresiva y poco frecuente, el cuadro clínico se caracteriza por espasticidad corticobulbar que inicia generalmente con afectación de las extremidades inferiores. El diagnóstico se basa en los criterios propuestos por Pringle et al., en 1992 (2).

En la neuropatología se puede evidenciar afectación selectiva de los haces corticoespinales aunque se reporta también compromiso de los haces espinocerebelosos. Generalmente esta afectación es bilateral y se extiende hasta la médula espinal, reflejándose estos hallazgos en los estudios de resonancia magnética nuclear (RMN) de encéfalo y de médula espinal (3).

La afectación del haz córticoespinal no es específica de EMN, ya que se puede encontrar en otras patologías como esclerosis múltiple, paraparesia espástica hereditaria (PEH), y otras entidades que entran en el diagnóstico diferencial de ELP (1), si bien la afección de los haces córticoespinales en estas entidades pueden ser simétricas, la mayoría de reportes coinciden en que la afectación generalmente es asimétrica, focal y poco extensa, alejando la posibilidad del hallazgo de imagen en "copa de vino", ya que esta imagen se configura cuando las lesiones de los haces corticoespinales son bilaterales, simétricas y extensas. Además a diferencia de ELP, otras entidades como la PHE no afectan selectivamente los haces corticosespinales o corticobulbares y presentan otras alteraciones como atrofia del cuerpo calloso y dilatación ventricular $(1,4)$.

Presentamos este caso para dar a conocer que, aunque infrecuente es posible la asociación de ELP y demencia frontotemporal (DFT), asimismo, para resaltar que la imagen en "copa de vino", puede estar presente en EMN y que particularmente asociada a ELP puede ser de gran ayuda en el diagnostico diferencial con otras entidades crónicas de curso clínicamente similar.

\section{Reporte de caso}

Varón de 56 años de edad, artesano ceramista, sin antecedentes familiares de importancia, que presenta un cuadro clínico de dos años de evolución, caracterizado por trastorno conductual progresivo, descuido del aseo personal, carácter irascible, lenguaje procaz, risa inmotivada e inapropiada al contexto social, apatía, desinterés por el trabajo, luego de un año de iniciado los síntomas, se agrega debilidad progresiva en miembros inferiores que le impedían la deambulación, y que posteriormente se extiende a miembros superiores.

Cinco meses previos a la hospitalización, se agrega disfagia para sólidos y líquidos, y diez días antes,

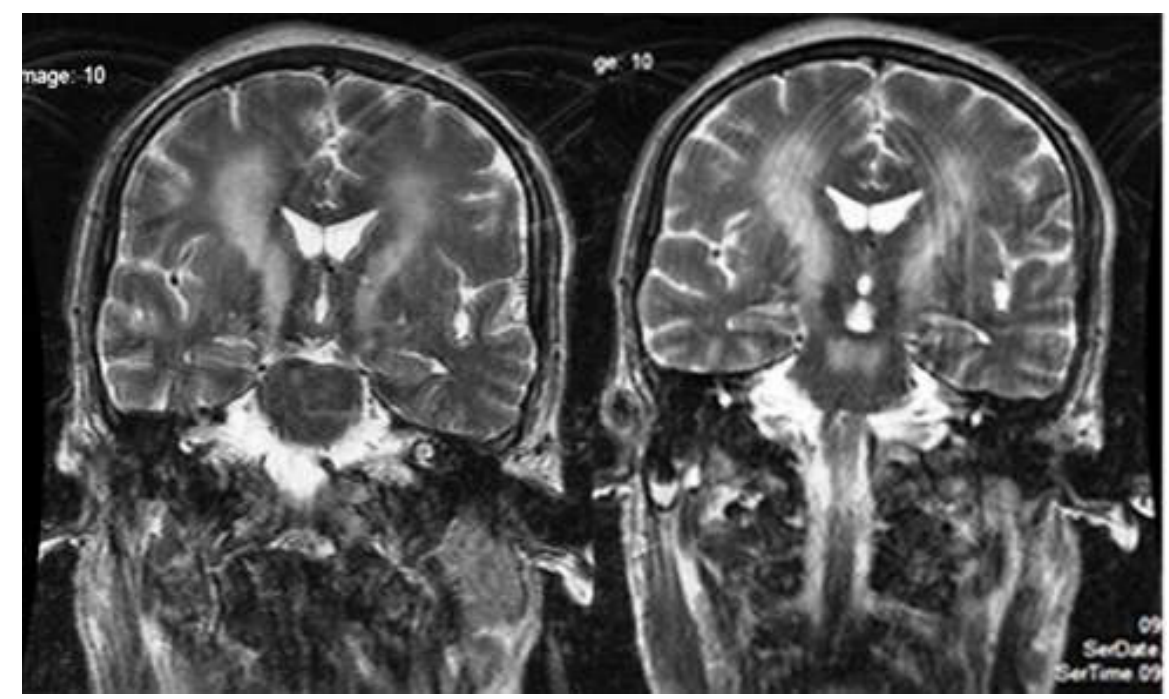

Figura 1. RMN secuencia T2 corte coronal: Hiperseñal que compromete bilateralmente los haces córtico espinales desde la corona radiada hasta puente. 
mostró desconexión del entorno, indiferencia afectiva, disartria, y disminución progresiva de fluencia verbal hasta el mutismo.

Al examen clínico paciente despierto, desorientado en tiempo y lugar, facies inexpresiva, afásico, no obedecía órdenes simples, presentó signos de liberación frontal (imitación, coleccionismo, prensión palmar, reflejo palmomentoniano y de succión), disminución del reflejo nauseoso con úvula central y velos simétricos, cuadriparesia espástica simétrica a predominio de miembros inferiores, Babinski bilateral y reflejos osteotendinosos conservados. No se evidenció fasciculaciones, ni atrofia muscular, la sensibilidad no fue evaluable.

Exámenes auxiliares: RPR (reagina plasmática rápida): no reactivo, HIV (virus de inmunodeficiencia humana): no reactivo, HTLV1 (virus linfotrófico humano ) en suero: no reactivo, hemograma (leucocitos 2300, hemoglobina $13.4 \mathrm{mg} / \mathrm{dl}$ ), LCR (líquido cefalorraquídeo): normal, Vitamina B12 >1200 pg/ $\mathrm{ml}$, ANCA (anticuerpos anticitoplasma de neutrófilos) y ANA ( anticuerpos antinucleares) negativos.

La electromiografía y velocidad a de conducción no evidenció signos de afectación de segunda motoneurona ni de nervio periférico.

La RMN de encéfalo en corte coronal reveló la presencia de hiperseñal que comprometía bilateralmente los haces corticoespinales desde el centro semioval hasta las pirámides bulbares dando la impresión de imagen en "copa de vino" (figura

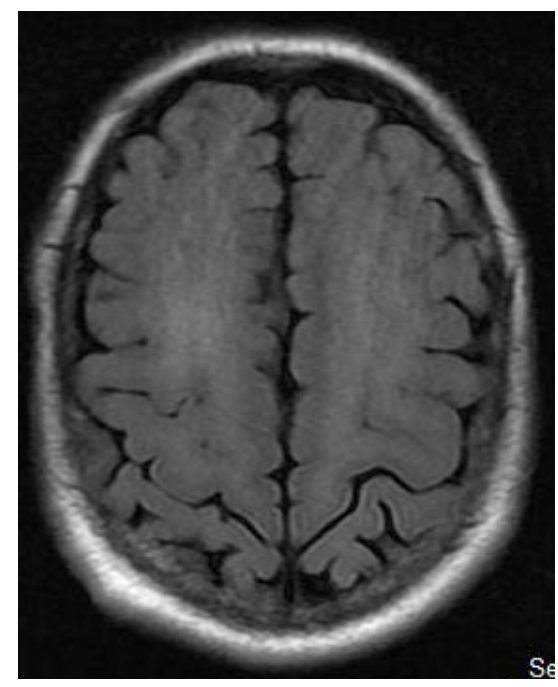

Figura 2. RMN secuencia Flair corte axial: Atrofia Fontal y Temporal bilateral a predominio izquierdo
1). Además, atrofia frontal y temporal bilateral a predominio izquierdo (figura 2) y disminución marcada del volumen del lobulillo precentral con acentuación del surco central.

La RMN no fue sugestiva ni mostro lesiones compatibles con esclerosis múltiple ni infartos lacunares incluidos en el diagnostico diferencial de la esclerosis lateral primaria.

Cabe señalar que no se realizó el estudio de HTLV1 en LCR, dosaje de anticuerpos antineuronales, ni de anticuerpos reumatológicos.

\section{DISCUSIÓN}

Nuestro paciente cumplió con los criterios propuestos por Pringle et al., para el diagnóstico de esclerosis lateral primaria (2).

El diagnostico diferencial fue amplio, sin embargo, el perfil clínico, imagenológico y los exámenes auxiliares nos ayudó a descartar la posibilidad de otros diagnósticos alternativos como infartos lacunares múltiples, enfermedades desmielinizantes, infección por virus de HTLV1, VIH, etc. Cabe mencionar que la causa de la elevación de la vitamina B12 observada en nuestro paciente, no pudo ser detectada, sin embargo, este hallazgo no aleja la posibilidad de ELP, ya que, si bien es cierto, los criterios de Pringle et al., exigen la normalidad de los niveles de vitamina B12, como apoyo diagnóstico (2), esta exigencia está más en relación con la posibilidad de confundirla con degeneración combinada subaguda asociada a la deficiencia de esta vitamina. (3)

El paciente previo a las manifestaciones motoras, presentó alteraciones conductuales marcadas con desinhibición y conducta social inapropiada y la presencia de signos de liberación frontal lo cual sugirió queprevioalcuadromotorel pacientepresentóuncuadro compatible con demencia frontotemporal. Aunque no se llegó a realizar la evaluación neuropsicológica formal por su estado, los datos imagenológicos y de anamnesis, en los que destacaban sus manifestaciones iniciales caracterizadas por trastorno conductual con signos y síntomas de liberación frontal sustentaban que el paciente cumplía los criterios diagnósticos propuestos por Rascovsky et al., para DFT variante conductual probable (5).

ELA ha sido considerado clásicamente un trastorno motor puro, en los últimos años se ha reconocido que 
Signo de la copa de vino: A propósito de un caso con esclerosis lateral primaria y demencia frontotemporal.

Tabla 1. Resumen de los casos publicados de la imagen en copa de vino asociados a ELA y ELP, comparando variables clínicas, demográficas y de Neuroimagen

\begin{tabular}{|c|c|c|c|c|c|c|c|}
\hline REFERENCIA & $\begin{array}{l}\text { EDAD / } \\
\text { SEXO / } \\
\text { TE }\end{array}$ & DIAGNÓSTICO & $\begin{array}{l}\text { DETERIORO } \\
\text { COGNITIVO/ } \\
\text { DEMENCIA }\end{array}$ & $\begin{array}{l}\text { ESTRUCTURAS } \\
\text { AFECTADAS }\end{array}$ & $\begin{array}{l}\text { EXTENSIÓN } \\
\text { AFECTACIÓN }\end{array}$ & $\begin{array}{l}\text { PATRON DE } \\
\text { AFECTACIÓN }\end{array}$ & $\begin{array}{l}\text { SIGNO } \\
\text { REPORTADO }\end{array}$ \\
\hline $\begin{array}{l}\text { Kuruwilla et al. } \\
2002\end{array}$ & $53^{\mathrm{a}} / \mathrm{M} / 3 \mathrm{a}$ & ELP & Ausente & Haz córtico espinal & $\begin{array}{l}\text { Puente-centro } \\
\text { semioval }\end{array}$ & $\begin{array}{l}\text { Bilateral/ } \\
\text { simétrico }\end{array}$ & Copa de vino \\
\hline $\begin{array}{l}\text { Banal et al., } \\
2009\end{array}$ & $37^{\mathrm{a}} / \mathrm{M} / \mathrm{NR}$ & ELA & NR & $\begin{array}{l}\text { Haz córtico } \\
\text { espinal+ cuerpo } \\
\text { calloso }\end{array}$ & $\begin{array}{l}\text { Médula } \\
\text { cervical-corona } \\
\text { radiada y cuerpo } \\
\text { calloso }\end{array}$ & $\begin{array}{l}\text { Bilateral/ } \\
\text { simétrico }\end{array}$ & Guirnalda \\
\hline $\begin{array}{l}\text { Parashary, et al. } \\
2011\end{array}$ & $40^{\mathrm{a}} / \mathrm{M} / \mathrm{NR}$ & ELA & NR & Haz córtico espinal & $\begin{array}{l}\text { Puente-centro } \\
\text { semioval }\end{array}$ & $\begin{array}{l}\text { Bilateral/ } \\
\text { simétrico }\end{array}$ & Copa de vino \\
\hline $\begin{array}{l}\text { Paliwal, et al. } \\
2012\end{array}$ & $45 / \mathrm{M} / 15 \mathrm{a}$ & ELP & Ausente & Haz córtico espinal & $\begin{array}{l}\text { Puente-Región } \\
\text { peri ventricular }\end{array}$ & $\begin{array}{l}\text { Bilateral- } \\
\text { simétrico }\end{array}$ & Copa de vino \\
\hline $\begin{array}{l}\text { Bazadona et al. } \\
2014\end{array}$ & $56^{\mathrm{a}} / \mathrm{F} / 9 \mathrm{~m}$ & ELA & NR & Haz córtico espinal & $\begin{array}{l}\text { Puente-peri } \\
\text { ventricular }\end{array}$ & $\begin{array}{l}\text { Bilateral/ } \\
\text { asimétrico }\end{array}$ & Copa de vino \\
\hline $\begin{array}{l}\text { Segura et al. } \\
2018\end{array}$ & $56^{\mathrm{a}} / \mathrm{M} / 2 \mathrm{a}$ & $\mathrm{ELP}+\mathrm{DFT}$ & Presente & Haz córtico espinal & $\begin{array}{l}\text { Puente-corona } \\
\text { radiada }\end{array}$ & $\begin{array}{l}\text { Bilateral/ } \\
\text { simétrico }\end{array}$ & Copa de vino \\
\hline
\end{tabular}

HCE: Haz córtico espinal; EMN: Enfermedad de motoneurona; ELP: Esclerosis lateral primaria; ELA: Esclerosis lateral amiotrófica

podría estar asociado a diversos grados de disfunción frontotemporal, cuyo grado extremo seria la DFT, configurando una entidad definida por Strong et al., como desorden del espectro ELA-DFT, al que incluso se le ha asignado criterios diagnósticos en los cuales todavía no se consideran las formas restringidas de enfermedad de motoneurona como la esclerosis lateral primaria o la atrofia muscular progresiva, aunque la tendencia es a considerarlas dentro de este espectro en el futuro (6).

ELA es la EMN más frecuentemente asociada con DFT, se sabe que $10-20 \%$ de los pacientes con ELA presentan además DFT. sin embargo, también se ha comunicado asociación de ELP y DFT en los cuales se demostró la presencia de cuerpos de inclusión de ubiquitina con presencia de TDP-43 en los estudios postmorten. Hallazgos anátomo patológicos que sustentan la relación de estas dos entidades $(7,8)$.

Los estudios anátomopatologicos muestran que la EMN cursa con degeneración progresiva del haz corticoespinal. Desde que Kojewnikoff en 1883, informó la presencia de degeneración de los tractos de sustancia blanca en un paciente con ELA (9), varios otros autores en los que destaca Holmes en 1909 han corroborado la degeneración selectiva del haz piramidal y la reducción del número de células piramidales de Betz en los pacientes con ELA aunque con diferente extensión y severidad (9).
Estos cambios pueden ser evidenciados en la resonancia magnética de los pacientes con EMN en forma de lesiones hiperintensas en las secuencias ponderadas en T2 que serían el reflejo de la degeneración Walleriana de estos haces (10).

Sin embargo, la expresión de la degeneración de los haces córtico espinales en RMN no es constante y cuando está presente, puede seguir diferentes patrones, puede ser bilateral o unilateral, focal o extenso y simétrico o asimétrico(11).

La mayoría de los reportes describen compromiso bilateral y simétrico de los haces córtico espinales, en los pacientes con EMN, sin embargo también se ha reportado compromiso focal y asimétrico restringido al centro semioval(1).

Si bien son ciertos los primeros reportes se hicieron en pacientes con ELA, como el reporte de Goodin et al., (9), posteriormente estos mismos hallazgos fueron observados en pacientes con $\operatorname{ELP}(1,12)$.

Mascalchi et al., comunicaron uno de los primeros casos de ELP con afectación bilateral y simétrica extensa de los haces corticoespinales (12).

Fabregas et al., observaron en RMN de encéfalo de imágenes de hiperseñal a lo largo de gran parte de la vía piramidal, en un paciente con criterios clínicos para ELP en el cual se confirmó posmorten, este diagnóstico 
con el hallazgo anatomopatológico de degeneración aislada del haz corticoespinal descendente se extendió hasta la médula espinal sin afectación de las células del asta anterior (10).

Kuruvilla et al., y Paliwal et al., en el 2012 comunicaron por primera vez la descripción en RMN de la imagen en copa de vino por afectación bilateral, simétrica y lineal de los haces corticoespinales observada en cortes coronales, ambos reportes se hicieron en pacientes con diagnóstico de ELP sin déficit cognitivo $(1,12)$. A diferencia de los casos reportados por estos autores nuestro paciente presentó un cuadro compatible con demencia frontotemporal asociada. (tabla 1)

Parashari et al., en el 2011, Bazadona et al., en el 2014 y Kumar et.al en el 2016 informaron la presencia del signo de la "copa de vino" asociado a ELA $(13,14,15)$, aunque en 2009 Bansal et al., ya lo habían hecho, pero como el signo de la "guirnalda" asociado a ELA (16).

Cabe señalar, que si bien es cierto varios autores antes de Kuruvilla ya habían comunicado la presencia de lesiones bilaterales, simétricas y extensas de los haces corticoespinales en RMN de encéfalo de los pacientes con EMN, ninguno de estos autores, mencionaron que las lesiones bilaterales de estos haces particularmente extensas podrían configurar una imagen con apariencia de "copa de vino" $(9,10,11)$ , esto pasó desapercibido debido probablemente a que sus descripciones se basaron en imágenes en cortes axiales y no en cortes coronales, en los cuales se observa esta configuración tan particular de las lesiones.

Cabe señalar que el signo de la copa de vino, también ha sido reportado asociado a cuadros de desmielinización osmótica, en correcciones bruscas de hipernatremia, sin embargo, estos cuadros se caracterizan por ser agudos o subagudos y distan del curso clínico con evolución crónica observada en EMN y particularmente en ELP. (17)

Finalmente podemos concluir que ELP también puede estar asociada con DFT, la cual es importante tenerla en cuenta, ya que tiene implicancia en el manejo de estos pacientes. Así mismo concluimos que la imagen en "copa de vino" es muy característica de ELP siendo particularmente útil para establecer su diagnóstico diferencial con otras entidades en el contexto de una patología crónica con disfunción piramidal y/o corticobulbar aislada en donde el diagnóstico diferencial puede ser un reto.

Contribución de autores: Darwin Segura-Chávez, Luis Torres Ramírez, Miriam Vélez Rojas y Martha Flores Mendoza; han contribuido en el diseño, redacción, aprobación final del manuscrito y asumimos la responsabilidad frente a todos los aspectos del manuscrito.

Fuente de financiamiento: Autofinanciado. Conflictos de interés: Los autores declaran no tener ningún conflicto de interés.

\section{Correspondencia}

Luis Torres Ramirez

Jr.Ancash 1271 Cercado de Lima, Perú.

Teléfono: 511-4117732

Correo electrónico: torresramirezl@hotmail.com

\section{REFERENCIAS BIBLIOGRÁFICAS}

1. Kuruvilla A, Joseph S. «Wine Glass» appearance : a unique MRI observation in a case of primary lateral sclerosis. Neurol India. 2002; 50 (3):306.

2. Pringle CE, Hudson AJ, Munoz DG, Kiernan JA, Brown WF, Ebers GC. Primary lateral sclerosis: Clinical features, neuropathology and diagnostic criteria. Brain J Neurol.1992; 115 (Pt 2):495-520.

3. Swash M, Desai J, Misra VP. What is primary lateral sclerosis? J Neurol Sci.1999; 170(1):5-10.

4. Raina S, Mokta JK, Sharma S. Hereditary spastic paraplegia with thin corpus callosum. Ann Indian Acad Neurol. 2009;12(1):56-7.

5. Rascovsky K, Hodges JR, Knopman D, Mendez MF, Kramer JH, Neuhaus J, et al. Sensitivity of revised diagnostic criteria for the behavioural variant of frontotemporal dementia. Brain. 2011;134(9):245677.

6. Strong MJ, Abrahams S, Goldstein LH, Woolley $\mathrm{S}$, Mclaughlin P, Snowden J, et al. Amyotrophic lateral sclerosis - frontotemporal spectrum disorder (ALS-FTSD): Revised. Amyotroph Lateral Scler Frontotemporal Degener. 2017; 18(3-4):153-174. doi: 10.1080/21678421.2016.1267768

7. Mackenzie IRA, Feldman H. Neurofilament inclusion body disease with early onset frontotemporal dementia and primary lateral sclerosis. Clin Neuropathol. 2004; 23(4):183-93.

8. Mochizuki A, Komatsuzaki Y, Iwamoto H, Shoji S. Frontotemporal dementia with ubiquitinated neuronal inclusions presenting with primary lateral sclerosis and parkinsonism: clinicopathological report of an autopsy case. Acta Neuropathol (Berl). 
Signo de la copa de vino: A propósito de un caso con esclerosis lateral primaria y demencia frontotemporal.

2004;107(4):377-80.

9. Goodin DS, Rowley HA, Olney RK. Magnetic resonance imaging in amyotrophic lateral sclerosis. Ann Neurol.1988; 23(4):418-20.

10. Martí-Fàbregas J, Pujol J. Selective involvement of the pyramidal tract on magnetic resonance imaging in primary lateral sclerosis. Neurology. 1990;40(11):1799-800.

11. Mascalchi M, Salvi F, Valzania F, Marcacci G, Bartolozzi C, Tassinari CA. Corticospinal tract degeneration in motor neuron disease. AJNR Am J Neuroradiol.1995; 16(Suppl 4):878-80.

12. Paliwal V, Rahi S, Singh P, Gupta R. «Wine-glass appearance» of pyramidal tracts in a patient with primary lateral sclerosis. Neurol India. 2012;60(1):110-1. doi: 10.4103/0028-3886.93606

13. Parashari U, Khanduri S, Bhadury S, Srivastava $\mathrm{D}$, Saxena $\mathrm{S}$. The magnetic resonance imaging «wine glass» sign of amyotrophic lateral sclerosis. Int J Nutr Pharmacol Neurol Dis. 2011; 1(2):206.
14. Bažadona D, Blažina K, Miletić V. 'Wine-glass' sign. Neurol Croat. 2014; 63(1):3-4.

15. Kumar S, Aga P, Gupta A, Kohli N. Juvenile amyotrophic lateral sclerosis: Classical wine glass sign on magnetic resonance imaging. J Pediatr Neurosci. 2016;11(1):56-7. doi: 10.4103/18171745.181251

16. Bansal AR, Dash GK, Radhakrishnan A, Kesavadas C, Nair M. «Garland sign» in amyotrophic lateral sclerosis. Neurol India. 2009; 57(3):354-5. doi: 10.4103/0028-3886.53273

17. Saroja AO, Naik KR, Mali RV, Kunam SR. 'Wine Glass' sign in recurrent postpartum hypernatremic osmotic cerebral demyelination. Ann Indian Acad Neurol. 2013;16(1):106-10. doi: 10.4103/09722327.107719

Recibido: 17/09/2018

Aceptado: 27/03/2019 